\title{
Editorial: Reactor Fuels, Materials and Systems Under Extreme Environments
}

\author{
Wenzhong Zhou ${ }^{1 *}$, Chunyu Zhang ${ }^{1}$, Shanfang Huang ${ }^{2}$, Zeyun $W^{3}{ }^{3}$ and Shripad T. Revankar ${ }^{4}$ \\ ${ }^{1}$ Sino-French Institute of Nuclear Engineering and Technology, Sun Yat-Sen University, Zhuhai, China, ${ }^{2}$ Department of \\ Engineering Physics, Tsinghua University, Beijing, China, ${ }^{3}$ Department of Mechanical and Nuclear Engineering, Virginia \\ Commonwealth University, Richmond, VA, United States, ${ }^{4}$ School of Nuclear Engineering, Purdue University, West Lafayette, IN, \\ United States
}

Keywords: experimental/computational fuels and materials, neutronics, thermal hydraulics, severe accident analysis, two-phase flow

\section{Editorial on the Research Topic}

Reactor Fuels, Materials and Systems Under Extreme Environments

\section{OPEN ACCESS}

Edited by: Wenxi Tian,

Xi'an Jiaotong University, China

Reviewed by:

Di Yun,

Xi'an Jiaotong University, China

*Correspondence:

Wenzhong Zhou

zhouwzh3@mail.sysu.edu.cn

Specialty section:

This article was submitted to

Nuclear Energy,

a section of the journal

Frontiers in Energy Research

Received: 23 January 2022

Accepted: 18 February 2022

Published: 04 March 2022

Citation:

Zhou W, Zhang C, Huang S, Wu Z and Revankar ST (2022) Editorial: Reactor Fuels, Materials and Systems Under

Extreme Environments.

Front. Energy Res. 10:860553. doi: 10.3389/fenrg.2022.860553
The safe and economical operation of any nuclear power system relies, to a great extent, on the success of the fuel and the materials of construction (Allen et al., 2010). Distinct from conventional power generation methods, a nuclear reactor is characterized by a harsh level of irradiation, very high operating temperature, and extremely corrosive environments. The behavior of fuels and materials as well as systems for fission reactors is thus complex due to the changes that the extreme environments generate on them. These kinds of changes may be seldom seen in other engineering disciplines, but this poses demanding requirements over reactor fuels, materials and systems. The design of reactor fuels, materials and systems subject to extreme environments is a major obstacle to enhancing reactor safety and economic performance of nuclear power systems. It is also essential in increasing innovation for current reactor operation improvement and future reactor design optimization. To address the challenges related to extreme reactor environments, the team of editors organizes the Research Topic "Reactor Fuels, Materials and Systems under Extreme Environments" in the journal Frontiers in Energy Research. Finally, 28 articles are collected, covering the theoretical, numerical and experimental studies on reactor fuels, materials and systems under extreme environments.

There are eleven articles about multiphysics/multiscale modeling and simulations of nuclear fuels and materials under extreme reactor environments, for light water reactors, liquid metal cooled fast reactors, and research reactors. In light water reactor studies, Zeng Z. et al. present three-dimensional modeling of thermal-mechanical behavior of accident tolerant fuels; Lu W. et al. deliver coupled modeling and simulation of phase transformation in zircaloy- 4 fuel cladding under loss-of-coolant accident conditions; Liu S. et al. work on multiphysics modeling of thorium-based fuel performance with $\mathrm{Cr}$-coated $\mathrm{SiC} / \mathrm{SiC}$ composite under normal and accident conditions; Yu M. et al. show multiphysics investigation on coolant thermohydraulic conditions and fuel rod behavior during a loss-of-coolant accident; Zhang X. et al. write 3dimensional multiphysics modeling of behaviors of pressurized water reactor fuel rods with missing pellet surface; Li X. et al. investigate hydrogen atom and molecule adsorptions on $\mathrm{FeCrAl}$ (100) surface by a firstprinciple study. In liquid metal cooled fast reactor investigations, Yuan $\mathrm{H}$. et al. contribute lead-cooled fast reactor annular UN fuel design and development of performance analysis program; Deng L. et al. write multiphysics model development for polonium transport behavior in a lead-cooled fast reactor. Wang G. et al. convey preliminary multiphysics performance analysis and design evaluation of $\mathrm{UO}_{2}$ fuel for LBEcooled subcritical reactor of China Initiative Accelerator Driven System. Meanwhile, in research reactor approaches, Mao X. et al. study the effects of U-Mo irradiation creep performance on the thermo- 
mechanical coupling behavior in U-Mo/Al monolithic fuel assemblies; Kong $\mathrm{X}$. et al. research on macro-mesoscale in-pile thermal-mechanical behavior simulation of a $\mathrm{UMo} / \mathrm{Zr}$ monolithic fuel plate.

Six articles focus on experimental accident tolerant fuels trying to overcome the challenges by the extreme reactor environments from the materials aspect. Ma X. et al. investigate elastoplastic deformation and fracture behavior of $\mathrm{Cr}$-coated $\mathrm{Zr}-4$ alloys for accident tolerant fuel claddings; $\mathrm{Ma} \mathrm{B}$. et al. study friction and wear properties of $\mathrm{CrAl}-$ based coatings for nuclear fuel cladding; Lai H. S. et al. work on the effect of rolling deformation on creep properties of FeCrAl alloys; Xiong Z. et al. present giga-cycle fatigue behavior of the nuclear structure of 316L weldments; Shi T. et al. contribute the formation of bubble-loop complexes during helium radiation in $\mathrm{Fe}-9 \mathrm{Cr}$ steel; Huang $\mathrm{X}$. et al. show their experimental investigation on the cleaning effect and influence rule of hydrogen peroxide-acetic acid on the lead-bismuth eutectic alloy.

Six articles emphasize reactor thermal hydraulics, severe accidents, and multiphase flow and heat transfer, which is vital in addressing extreme reactor conditions. Liu Y. et al. present their experimental measurement on pebble flow discharge in a hopper silo based on a drainage method; Hao Y. et al. deliver a study on the calculation method of molten pool decay heat distribution under IVR condition. $\mathrm{Hu} \mathrm{P}$. and $\mathrm{Hu} \mathrm{Z}$. contribute a numerical study of the impingement of water film on a small attached bulging plate on a vertical plane; Di Ronco et al. theoretically work on an Eulerian single-phase transport model for solid fission products in the molten salt fast reactor to develop an analytical solution for verification purposes; Salmassian B. et al. perform a transient parameter analysis of non-scrammed local melting accidents with a VVER 1000 case study; Yang T.

\section{REFERENCE}

Allen, T., Busby, J., Meyer, M., and Petti, D. (2010). Materials Challenges for Nuclear Systems. Mater. Today. 13, 14-23. doi:10.1016/s1369-7021(10)70220-0

Conflict of Interest: The authors declare that the research was conducted in the absence of any commercial or financial relationships that could be construed as a potential conflict of interest.

Publisher's Note: All claims expressed in this article are solely those of the authors and do not necessarily represent those of their affiliated et al. convey a study on thermophysical properties of a lead-bismuth-based graphene nanofluid.

Neutronics and reactor engineering analysis under extreme environments includes three articles. Düz M. presents neutronic calculations for certain Americium mixed fuels and clads in a boiling water reactor; $\mathrm{Li} \mathrm{H}$. et al. introduce an approach to optimize the RMC code Using the decay chain method for large-scale decay calculations; Cai $\mathrm{B}$. et al. present a neutronic design for heat pipe reactor with annular and accident tolerant fuels.

Finally, this research topic also includes two review articles to overview state-of-the-art research approaches on extreme reactor environments. $\mathrm{Li} \mathrm{K}$. et al. deliver a review on $\mathrm{BeO}$ utilization in reactors for the improvement of extreme reactor environments; Guo J. et al. contribute a review of models and experiments on fission gas behaviors and relevant phenomena in different nuclear fuels.

After almost 1 year's revisions and updates, this research topic finally selected those 28 articles and published them in the journal Frontiers in Energy Research. The collection shows the ongoing research on reactor fuels, materials and systems under extreme environments from different perspectives, and also allows us to exhibit the high-quality work to the public. More high-quality topics and articles are welcomed at this open platform in the future. All the editors are open to further information and communications.

\section{AUTHOR CONTRIBUTIONS}

All authors listed have made a substantial, direct, and intellectual contribution to the work and approved it for publication.

organizations, or those of the publisher, the editors and the reviewers. Any product that may be evaluated in this article, or claim that may be made by its manufacturer, is not guaranteed or endorsed by the publisher.

Copyright (C) 2022 Zhou, Zhang, Huang, Wu and Revankar. This is an open-access article distributed under the terms of the Creative Commons Attribution License (CC $B Y$ ). The use, distribution or reproduction in other forums is permitted, provided the original author(s) and the copyright owner(s) are credited and that the original publication in this journal is cited, in accordance with accepted academic practice. No use, distribution or reproduction is permitted which does not comply with these terms. 